\title{
Do true preretinal (subhyaloid) hemorrhages occur in infants?
}

\author{
Horace B. Gardner
}

Received: 19 October 2011 / Accepted: 8 November 2011 /Published online: 19 November 2011

(C) Springer-Verlag 2011

The recent educational paper by Taylor [4] uses the ambiguous descriptor "preretinal" to describe intraocular hemorrhages. Clinically, this term has been used to describe both "true" preretinal (subhyaloid) hemorrhages and subinternal limiting membrane (subILM) hemorrhages. Emerson et al. [1] uses neither subhyaloid nor preretinal in his pathological description of intraocular hemorrhage and indeed finds the blood to be subILM in all 41 of his cases with macular hemorrhage. Why this is so may be explained by Sebag's [2] demonstration of a "sheetlike" adherence of the vitreous that encompasses the macula as well as the peripapillary posterior pole in individuals 20 years of age or younger. Thus, although as Srinivasan and Kyle's [3] picture of a subhyaloid hemorrhage in the macula overlying a subILM hemorrhage in the same eye in a 46 year old proves hemorrhage can occur in either space in an adult, it is possible that in infants the adhesive force between the vitreous and ILM exceeds the cohesive force of the vitreous and thus the blood cannot dissect between the vitreous and the ILM but instead breaks into the vitreous as seen in almost $40 \%$ of Emerson's cases.

Taylor also references a piglet study by Coats et al. This study used rotational accelerations over 50 times those achieved by human shaking yet produced only microscopic retinal hemorrhage at the retina/ciliary body junction and anterior chamber hemorrhage (hyphema) and ciliary body disruption. These are findings associated with direct eye trauma. They are not the extensive posterior retinal hemorrhage associated with abusive head trauma. Thus, this study would suggest that the eye findings of abusive head trauma are not produced by rotational acceleration.

Conflict of interest The author Horace Gardner testifies in presumed abusive head trauma cases. He does not believe this creates any conflict of interest.

\section{References}

1. Emerson M, Jakobs E, Green W (2007) Ocular autopsy and histopathologic features of child abuse. Ophthalmology 114:1384-1394

2. Sebag J (1991) Age related differences in the human vitreoretinal interface. Arch Ophthalmol 109:966-971

3. Srinivasan S, Kyle G (2006) Subinternal limiting membrane and subhyaloid haemorrhage in Terson syndrome: the macular 'double' ring sign. Eye 20:1099-1101

4. Taylor D (2011) Retinal haemorrhages in abusive head trauma in children. Eur J Pediatr. doi:10.1007/s00431-011-1579-2
H. B. Gardner $(\bowtie)$

318 Oklahoma Road,

Manitou Springs, CO, USA

e-mail: horacebgardner@yahoo.com 